could safely remove worn out or defective structural parts, build new entrances and exits, and that without disturbing unduly the regular routine of activities going on in the building.

But when he ceased to be contented with walls and partitions, and went into the kitchen, the laundry, the toilet, and the cellar, he soon found that his old set of rules would not apply. The action of vital forces has not yet been definitely reduced to a simple working formula. To solve any problem there must be as many equations as unknown quantities. The trouble seems to be that in the present discussion the $X$ 's and Y's and Z's are in excess.

When the first gastroenterostomies gave immediate relief to the hunger-pains of the man with duodenal ulcer, a new miracle seemed to have been wrought. But the "vicious circle" drove out the anterior operation and brought in the posterior direct attachment and the anastomosis "in Y," and now it is found that the real stomach ulcer, situated at a distance. from the pylorus, and the second jejunal one, remain to trouble us.

Mr. Lane, with smiling insouciance, cuts and trims and splices, but many have found, to their sorrow, that in their hand at least the problem is something more than a mere plumber's job of wiping a joint.

Meanwhile we, who are on the back seats, looking to you on the floor of the arena, are diligently and hopefully watching your results, believing that in good time you will be able to point out for us where lies the path of safety and success for our patients and ourselves.

\section{(Orininal Artixleg.}

\section{HEMORRHAGIC OSTEOMYELITIS.}

\section{By Ernst H. Arnold, M.D., New Haven, Conn.}

Several reasons prompt me to bring to your attention the above subject at the present time. Hemorrhagic osteomyelitis is apt to exist without symptoms subjective or objective and may go, therefore, unrecognized. Fracture without what would be to a healthy bone sufficient provocation may take place. A bone having thus been broken and hemorrhagic osteomyelitis yet not recognized as the remote causative agent, delayed or non-union may. result.

If the lesion be situated near a joint which from the predilection of the lesion for spongy bone is likely to be the case, it may be mistaken for a joint lesion such as tuberculosis. Conservative treatment for the latter, braces for instance, have no influence on hemorrhagic osteomyelitis, whereas prompt surgical interference would speedily restitute the part $a d$ integram. With the former the patient would face a long drawn out and inefficient course of treatment.
Finally, hemorrhagic osteomyelitis may be mistaken for a malignant bone lesion and drastic and mutilating surgical measures taken which would be altogether unnecessary.

In view of these reasons a recital of the nature and behavior of this condition together with the relation of a few illustrative cases may not be out of place.

Etiology. The condition is not very frequent. This infrequency is in part undoubtedly due to the fact that the lesion exists often unrecognized and undiagnosed. Its preference for spongy bone explains its occurrence in childhood and youth. I myself have not come across a case older than twenty-five years. The pathology being that of a process of repair, the injury to the tissue calling for repair must, in the absence of infection, be traumatic. That seems to be the case history as the history of trauma is frequently given.

Pathology. The gross pathology of the lesion usually presents a cavity in the spongy part of a long bone. When the formation of this cavity has progressed for some time it consists of one chamber of very regular, often oval outline. If bone absorption has not yet advanced much, the unabsorbed trabeculae may subdivide this chamber in several compartments. The cavity is found filled with a dark brownish mass of jelly-like consistency from which uncoagulated blood oozes. A membrane, brownish red in appearance, fairly thick, one-eighth inch or more, and which is very friable, lines the cavity. The cavity expands, driving the cortica before it, but unless there be spontaneous fracture, rarely ruptures through it. The lesion is noticed then as a swelling of regular shape, and smooth surface. There are no signs of inflammation, no reddening, no heat till the pressure from underneath causes the skin reaction to show these signs. Microscopically, the mass is found to be a hemorrhagic extravasation in which light colored areas consisting of young fibroblastic tissue are noticed. In these are found many multinucleated giantcells of the foreign-body type of cell. They are derived from the fusion of leukocytes proliferated from vascular endothelium. The giant-cell formation is due to the presence of much hemorrhagic granular tissue. It is also favored by the necrosis in the extravasated areas. The tissue necrosis is likewise responsible for the appearance of fat globules in and around the endothelium cells. Numerous fibroblasts are seen forming collagen fibrils. Where the process becomes chronic this collagen formation is well marked, proceeding to fibrous contraction and cyst formation. This cyst formation and the presence of giant-cells were undoubtedly responsible for the view held in many quarters until quite recently, that the condition was one of tumor formation, bone cysts of this variety being classed as giant-cell sarcoma. However, it is now recognized that the process is one of a low 
grade inflammation usually chronic in character in response to trauma. The giant-cells have not a tumor forming but as Barrie, who has done a great deal of excellent work on this condition, puts it, scavenger function.

Symptoms. There may not be any subjective symptoms. Pain is present only when the expansion has gone far enough to make the cortical support of ligament or muscle attachment insufficient. We have then, pain on joint movement in the first, or muscle contraction in the latter case. Likewise may there be absence of objective symptoms and the condition is only accidentally revealed either when $x$-rays are employed for other conditions in the neighborhood of the lesion or by the fracture of the bone. With the expansion, however, of the lesion, we have a distinct enlargement of the bone. Usually there is neither heat nor redness to be noted. Only when the pressure from below becomes keen do these set in. There may be enlarged superficial veins over the swelling due to pressure interference with the venous circulation. In the lower extremity limping may be associated with the condition. The condition being strictly a local one, no general symptoms such as fever, anemia or loss of weight need be looked for. Atrophy of the soft parts in the neighborhood is absent in the beginning and not particularly marked in the advanced states. If the lesion is very near the joint there is some limitation of movement but passive movement within the limitation is painless. Protective muscle spasms are absent or only slightly marked.

Diagnosis. The youth of the patient, the location in spongy bone, the history of trauma, the absence of marked pain, limitation of movement and muscle spasm, the long duration without exacerbations or metastasis make the diagnosis likely. The x-ray findings are sufficiently characteristic to confirm the tentative clinical diagnosis.

The x-ray shows a cavity usually very regular in shape, sharply outlined. The cavity shows no bone proliferation. The cortica, unless broken by violence, is intact. If broken, no bony tumor mass is shown outside the cortica.

The differential diagnosis is important in re. gard to malignant bone tumor (sarcoma) and bone tuberculosis. Hemorrhagic osteomyelitis has the age of the patient in common with both. The location is the same in tuberculosis and may be the same in sarcoma.

The history of trauma may be had in all three. The presence of early and great pain distinguishes tuberculosis and sarcoma from it. Limitation of movement is an early and constant symptom of tuberculosis; it is marked in sarcoma near joints. Muscle spasms are marked and constant in tuberculosis and sarcoma near the joint. The local swelling of tuberculosis is diffuse and involves the soft parts. In sarcoma it may be circumscript but is irregular. The tubercular joint is hot. Sarcoma and hemor-

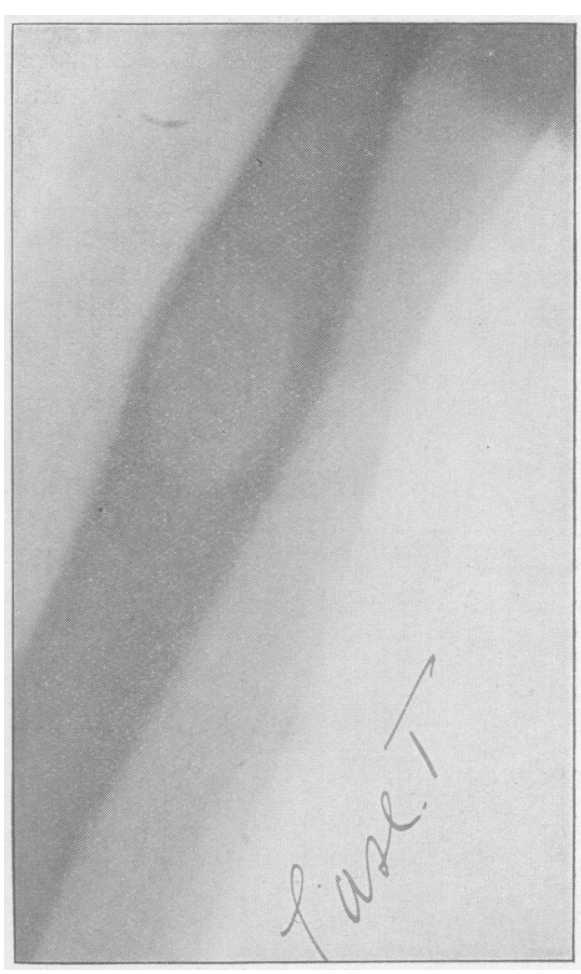

CASE I.

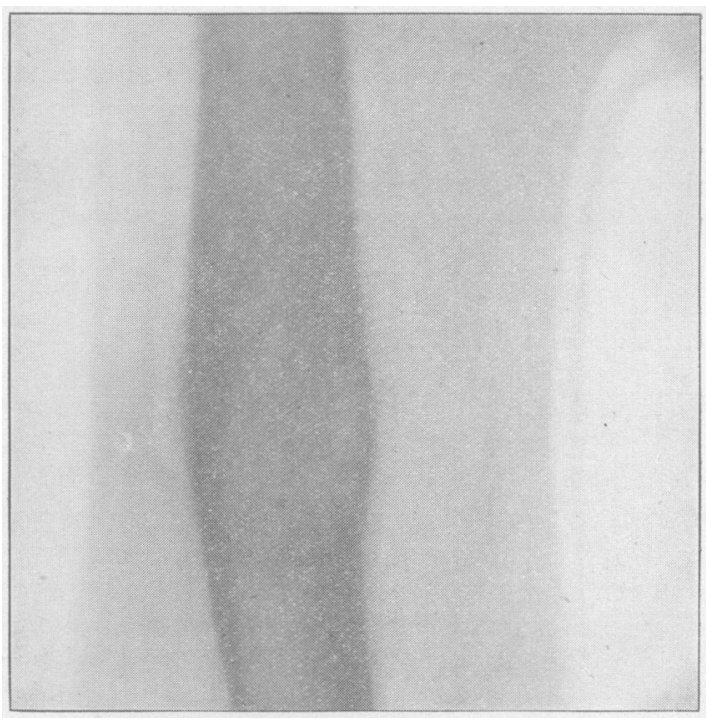

OASE I.

rhagic osteomyelitis only show redness and heat when the skin becomes involved. Tuberculosis and sarcoma are progressive; hemorrhagic osteomyelitis may be stationary for years.

Atrophy of soft parts adjacent above and below the lesion is present in tuberculosis and sarcoma. It is absent in hemorrhagic osteomyelitis except where long disuse has brought it about. No fever or general marasmus is noticed in hemorrhagic osteomyelitis. The x-ray usually clinches the matter. Tuberculosis presents no cavity, sarcoma not one of such regular shape and smooth outline. Sarenma involves the cor- 


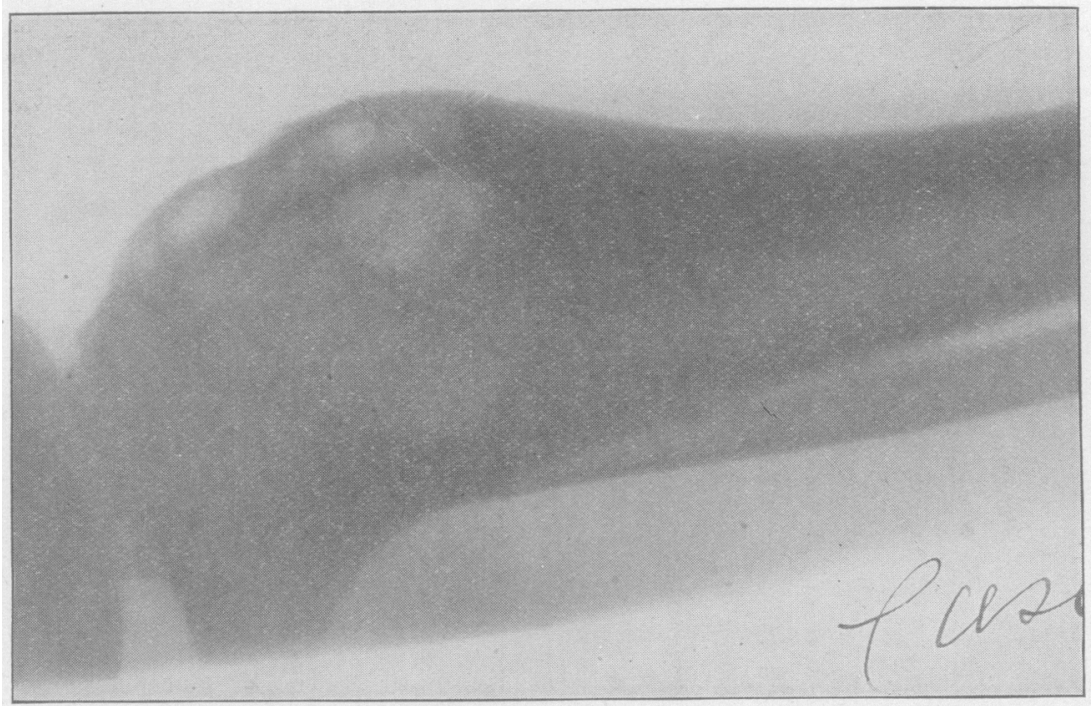

CASE II.

tica, breaks through it and usually shows bony mass outside of it.

Treatment. A sufficient opening through the cortica to allow the complete curettage of the hemorrhagic membrane and of the whole cavity and recesses, if there be such. If the cavity is large, filling it with bone chips taken from adjacent healthy cortica is helpful. Suture of periosteum and skin. No drainage. No use of tissue destroying swabbing such as carbolic acid. No bone wax filling. Perhaps aft a mildly stimulating application of dilute pat tincture of iodine may be had. If the cavity sign be large and the cortica thin, some protecting splint must be worn even in the upper extremity. In the lower extremity a protecting and supporting brace is indispensable till the $x$-ray shows sufficient bone deposit in the cavity to prevent untoward accidents. The x-ray taken at fairly frequent intervals (six to eight weeks) will control the recovery and indicate when protection may be done away with. The prognosis is uniformly good.

\section{HISTORY OF ILLUSTRATIVE CASES.}

CASE 1. Dates back to January, 1909, and is my earliest acruaintance with the condition. The patient, male, ten years, in poor health and flesh, injured left-upper arm several days ago. The injury was held to be a fracture by the attending physician. On examination, a distinct lump was felt between the middle and upper third of humerus. This was thought to be due to over-riding of fragments. The personal history related several injuries to the same arm previous to the present one; the last one about ten months ago. They were not, however, held to be fractures at the time. The x-ray revealed the lump to be an atrophic bone lesion, oval in shape and evidently cystic in nature, involving the medulla of the humerus. The appearance suggested a neoplasm which the history of the repeated injuries made likely. Exploratory operation was advised and done the same month, the plan being to be prepared to amputate should the gross appearance and fresh section examination show malignancy beyond doubt. At operation a cavity was found containing a blood-like mass and a hemorrhagic membrane. Specimens of these and bone chips from the wall of the cavity were taken for examination. There being no distinet sign of neoplasm the wound was closed after curettage and the arm splinted. The pathologic findings of Dr. Bartlett revealed no sign of malignant tumor formation but suggested a low grade chronic inflammation. A slow but apparently complete recovery took place. However, the arm was broken again, with little provocation, in the same place, in October of the same year. It was treated by splinting, after union, for some time by stimulating treatment, such as heat and massage. The patient is well today, the arm has completely recovered and no further difficulties with it have been experienced.

CASE 2. Connects the lesion directly with injury. Patient, female, 24 years, seen January, 1920. In September, 1919, she fell, striking the front of the tibia below the knee on the edge of a trolley rail. The bruise promptly healed, but some time after a swelling was noticed at the site of the former injury. At the time of the first examination this swelling was about the size of a hen's egg, smooth to touch. The skin over it showed some pressure signs. The $\mathrm{x}$-ray presented a cystic cavity with several compartments. The diagnosis of osteomyelitis hemorrhagica was made, and operation done. The cavity was filled with a gelatinous mass and lined by a hemorrhagic membrane. It was curetted and closed. A protective brace was worn for six months, a complete recovery was made, the patient is now well and about. 


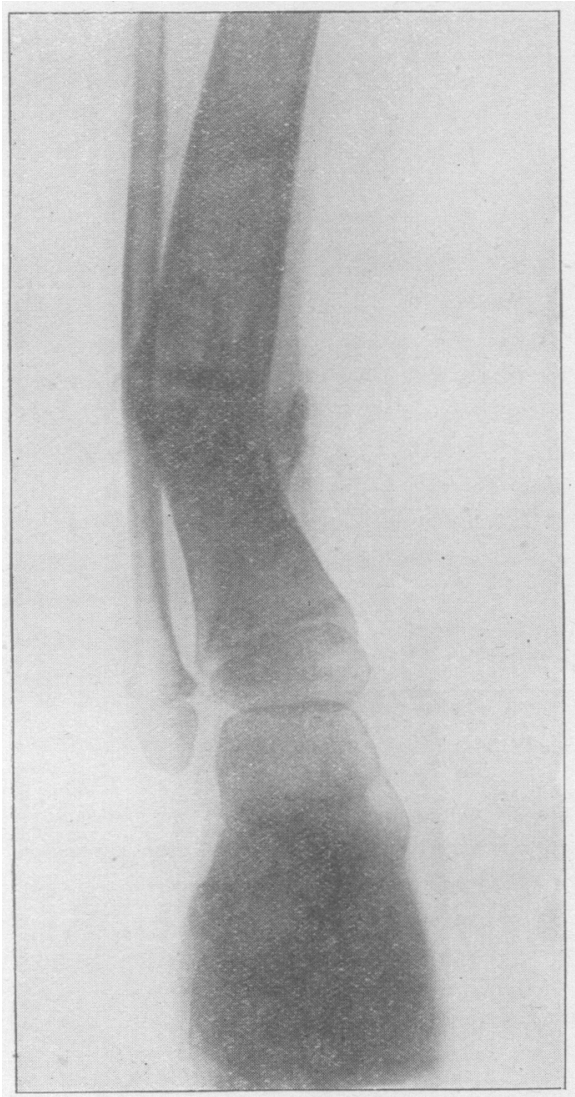

CA I III

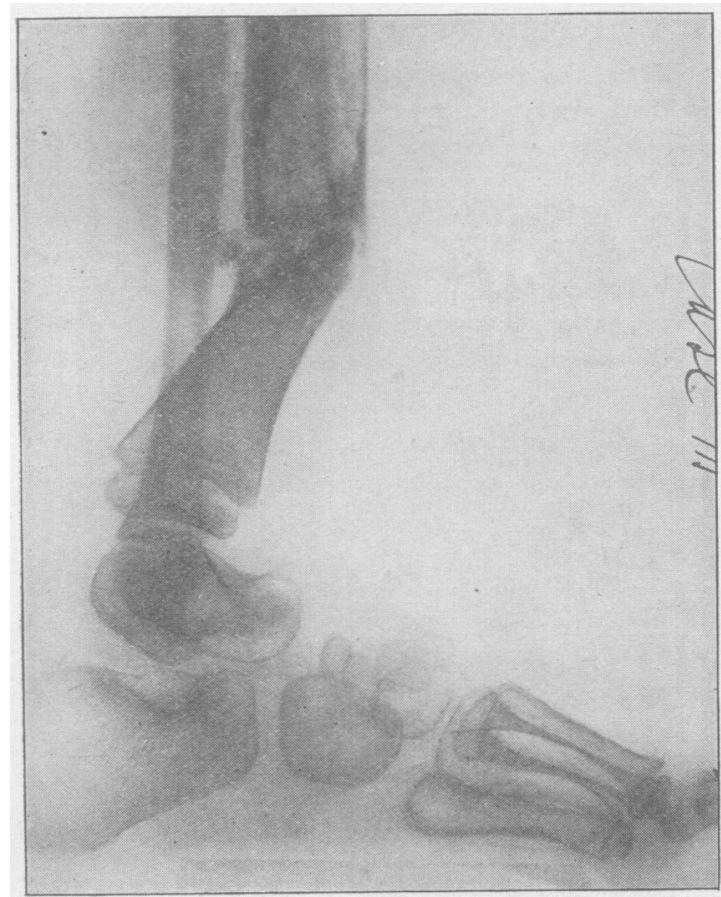

ARNOLD CASE IIL

CaSE 3. Seen June, 1920, has also the history of trauma, but whether the osteomyelitis found at the time of the first $x$-ray examination was the cause or effect of the injury cannot be

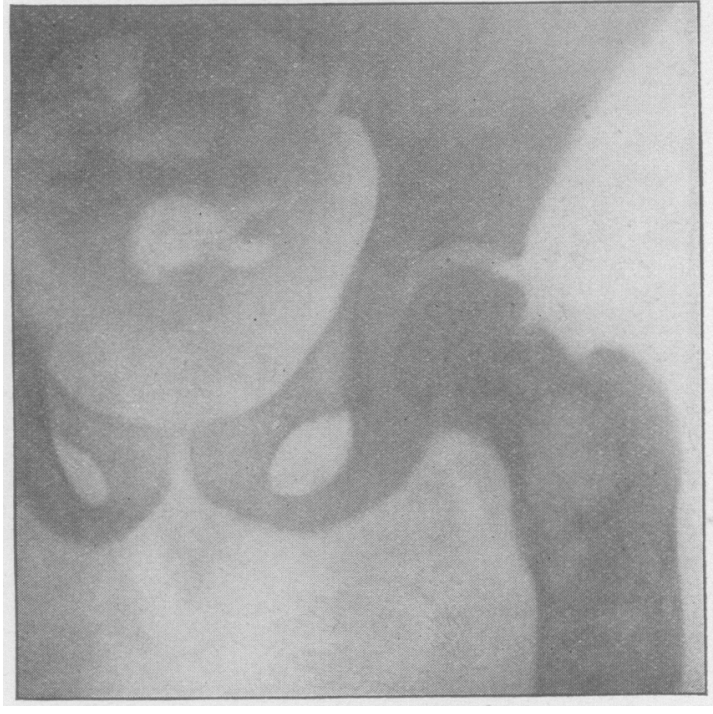

CASE IV.

told. Patient, male, five years, fell breaking leg, June, 1919. Since then the patient has been seen by quite a number of practitioners. He wears a protective brace but he limps with and without it, considerably. The tibia is bent to an angle of 150 degrees and there is a false point of motion. The x-ray shows an ununited fracture and bone cysts in the proximal fragment. The diagnosis--ununited fracture in presence of osteomyelitis hemorrhagica. At operation the lower fragment has its edges freshened. The upper fragment is found to have a cavity containing a bloody mass and a hemorrhagic membrane. This is curetted out. Opposition of fragments without suture, cast applied for six weeks, bony union promptly followed. $A$ protective brace was worn, the patient is well and about, and the union firm.

CASE 4. Illustrative of the possibility of lesion being mistaken for a tubercular joint. $\mathrm{Pa}$ tient, female, six years, in fair health and flesh, has been lame in left leg for upward of six months. There has been oceasional pain, but not severe. There is no atrophy of the muscles of the thigh or gluteofemoral region, only rotation in hip is limited. The x-ray shows a large cystic area involving the region in the neck, trochanter and upper part of shaft. Diagnosis of osteomyelitis hemorrhagica was made. This diagnosis is confirmed by operation, the lesion being entered through the trochanter. A large cavity filled with a bloody mass and lined by a hemorrhagic membrane is exposed. Curettage was done. A supporting brace is worn and should be worn for some time for fear of fracture through the very thin bony shell remaining after curettage. The operation is followed by the cessation of pain and the limitation of movement. The lesion is filling in. The patient is well and about, though still, for above cited reason, on a supporting brace. 